\title{
Fast Detection And Quantification of Plasmodium Species Infected Erythrocytes In A Non-Endemic Region By Using The Sysmex XN-31 Analyzer
}

\section{T.A. Khartabil ( $\sim$ t.khartabil@erasmusmc.nl )}

Erasmus MC University Medical Center https://orcid.org/0000-0002-7083-1710

Y.B. de Rijke

Erasmus MC University Medical Center

R. Koelewijn

Erasmus MC University Medical Center

J.J. van Hellemond

Erasmus MC University Medical Center

H. Russcher

Erasmus MC University Medical Center

\section{Research Article}

Keywords: Malaria, diagnosis, flow cytometry, hemocytometry, Plasmodium

Posted Date: December 21st, 2021

DOI: https://doi.org/10.21203/rs.3.rs-1145139/v1

License: (9) This work is licensed under a Creative Commons Attribution 4.0 International License. Read Full License

Version of Record: A version of this preprint was published at Malaria Journal on April 11th, 2022. See the published version at https://doi.org/10.1186/s12936-022-04147-0. 
1 TITLE PAGE

2

3

4

5 Fast detection and quantification of Plasmodium species infected erythrocytes in a nonendemic region by using the Sysmex XN-31 analyzer

7 T.A. Khartabil ${ }^{1}$, Y.B. de Rijke ${ }^{1}$, R. Koelewijn ${ }^{2}$, J.J. van Hellemond ${ }^{2}$, H. Russcher ${ }^{1}$ 8

$9{ }^{1}$ Department of Clinical Chemistry, Erasmus MC University Medical Center, Rotterdam, the

$10 \quad$ Netherlands

$11{ }^{2}$ Department of Medical Microbiology and Infectious Diseases, Erasmus MC University Medical

12 Center, Rotterdam, the Netherlands

13

14

15

16

17

18

19

20

21 Corresponding Author:

22 Henk Russcher

23 Dr. Molewaterplein 40

243015 GD Rotterdam

25 h.russcher@erasmusmc.nl 


\section{ABSTRACT}

27 BACKGOUND: Due to increased travel from endemic countries, malaria occurs more frequently

28 in non-endemic regions. It is a challenge for diagnostic laboratories in non-endemic countries to

29 provide reliable results, as experience of staff is often limited to only a few cases per year. In this

30 study, we evaluated the diagnostic accuracy of the fully automated Sysmex XN-31 malaria

31 analyzer in a routine diagnostic setting in a non-endemic region.

32 METHODS: Samples from 112 patients suspected for malaria were examined by the Sysmex XN-

3331 analyzer to determine the absolute count of malaria-infected red blood cells count (MI-RBC/ $\mu \mathrm{l})$.

34 Microscopic examination of both Quantitative Blood Coat capillary tubes and thick and thin blood

35 films were used as reference methods. Limits of blank (LoB), detection (LoD) and quantification

36 (LoQ) were investigated using an in vitro Plasmodium falciparum culture. Nine hundred twenty

37 samples of patients with RBC abnormalities were included to determine which RBC abnormalities

38 trigger indeterminate or false positive results.

39 RESULTS: No false positive nor false negative results were obtained for the examined patient

40 samples suspected for malaria. For 3\% of samples an indeterminate result by the XN-31 was

41 obtained. The Passing-Bablok regression line for diagnostic accuracy of the parasitemia was $\mathrm{y}=$

$4239.75+0.7892 x$ showing a positive bias of about $21 \%$ when comparing the MI-RBC results to

43 microscopy. The LoB, LoD and LoQ were calculated to be 4.7, 5.9, and 19.0 infected RBC/ $\mu \mathrm{L}$,

44 respectively. From the 920 abnormal RBC samples collected, $4.6 \%$ resulted in a false positive MI-

45 RBC result and almost half of the samples produced indeterminate results. These results were

46 related to increases in nucleated red blood cells, reticulocytes and other abnormal RBC

47 morphologies such as sickle cells. 
48 CONCLUSIONS: Based on the results we conclude that the $\mathrm{XN}-31$ is a fast and reliable screening

49 method in the detection and quantification of Plasmodium species in patients However, if an

50 abnormal red blood cell morphology is present, the results of the XN-31 should be interpreted with

51 caution as false positive results can be caused by interfering abnormal erythrocytes.

52 Keywords: Malaria, diagnosis, flow cytometry, hemocytometry, Plasmodium

\section{INTRODUCTION}

55 Malaria is a life-threatening disease caused by the protozoan parasite Plasmodium, which is

56 transmitted through the bite of an infected Anopheles mosquito. In 2019 there were 229 million

57 infections with 409,000 deaths reported globally. ${ }^{1}$ Most of these cases occur in sub-Saharan Africa,

58 which is responsible for $93 \%$ of cases and $94 \%$ of deaths. ${ }^{1}$ Due to the increasing number of global

59 travelers and immigration from endemic countries malaria becomes more relevant in non-endemic

60 countries in moderate climates as well. The five common Plasmodium species known to infect

61 humans are $P$. falciparum, $P$. vivax, $P$. ovale, $P$. malariae and $P$. knowlesi. $P$. falciparum causes

62 more than $90 \%$ of malaria cases and also causes the most fulminant and possibly fatal disease. ${ }^{2}$

64 Early and accurate diagnosis of malaria is fundamental for successful and timely treatment of the 65 disease, as delay and/or misdiagnosis can result in morbidity and mortality. According to the 66 World Health Organization (WHO), it is recommended to have prompt malaria diagnosis either

67 by microscopy or by a malaria rapid diagnostic test (RDT) in all patients with suspected malaria

68 before treatment is administered. ${ }^{3}$ Microscopic examination of thick and thin blood films remains

69 the golden standard according to current CDC guidelines. ${ }^{4}$ This method, however, is time

70 consuming and requires the availability of an experienced microscopist to examine the blood films, 
71 which is often a challenge outside office hours and significant variability in applied methods exist

72 among laboratories. ${ }^{5}$ Therefore, RDTs for malaria have been developed of which

73 immunochromatographic card tests (ICT) that detect antigens of Plasmodium in blood of the host,

74 are most commonly used. These ICTs are easy to perform and provide results within 10-15

75 minutes. ${ }^{6}$ However in case of infections with a low parasitemia, false negative results can be

76 obtained. ${ }^{7}$ Furthermore, the sensitivity of RDT testing is decreasing for the detection of $P$.

77 falciparum due to specific mutations in, or complete deletion of, the HRP-2 gene of P. falciparum. ${ }^{8}$

78 Alternative methods like Loop Mediated Isothermal Amplification (LAMP), Real-Time PCR (rt-

79 PCR) and Quantitative Buffy Coat (QBC) fluorescence analysis can be used as reliable alternative

80 screening methods, but are either time consuming, expensive and/or in need of trained staff to be

81 available 24 hours a day, seven days a week..$^{5-9-11}$ Hence, no ideal laboratory method is currently

82 available that provides fast and reliable results with information on both the Plasmodium species

83 (as these can require different therapy) and the parasitemia value needed to assess the disease

84 severity of patients infected with P. falciparum or P. knowlesi) without the need of well-trained

85 technicians. This is especially relevant for diagnostic laboratories in non-endemic countries,

86 because these encounter often only a few malaria cases per year and thus lack experience to

87 diagnose malaria by microscopic examination of blood films. ${ }^{5}$

89 The Sysmex XN-31 hemocytometer is an automated analyzer launched in September 2019 to

90 support malaria diagnosis in whole blood samples in the clinical diagnostic laboratory. Using

91 fluorescence flow cytometry (FFC) technology and a violet semiconductor laser with a $405 \mathrm{~nm}$

92 wavelength, this hemocytometer can detect, specify and quantitate malaria-infected red blood cells

93 (MI-RBC) within a specific area of the scattergram known as the M-gating area. Previous studies 
94 on the $\mathrm{XN}-31$ and its predecessor the $\mathrm{XN}-30$ reported mainly data on $P$. falciparum infected 95 patients in endemic countries ${ }^{12-16}$ or on in vitro cultures ${ }^{13,17,18}$. The XN-30, was approved for 96 research purposes only, whereas the $\mathrm{XN}-31$ is a CE marked in vitro diagnostic device with identical

97 hardware, software, and user interface. The XN-31 has not been evaluated in clinical practice in 98 non-endemic countries nor for Plasmodium species other than P. falciparum and P. vivax. ${ }^{13,14,16 .}$

99 In this study we compared the performance of the $\mathrm{XN}-31$ in clinical practice with the current 100 diagnostic workflow in our hospital, which is based on ICT in combination with microscopy by 101 QBC analysis and thin and thick blood film examination. In addition, the limit of blank (LoB), 102 limit of detection (LoD), and the limit of quantification (LoQ) were determined and compared to 103 previous values reported by Sysmex in the Instructions for Use. Since the XN-31 can report 104 indeterminate results that have been suggested to be linked to interference caused by certain RBC 105 abnormalities, such as are commonly observed in peripheral blood smears of patients with sickle 106 cell disease or other hemoglobinopathies. ${ }^{12}$ We investigated the specificity of Plasmodium infected 107 RBC detection by the XN-31, by examining 920 blood samples derived from patients with a wide 108 array of RBC abnormalities.

\section{METHODS}

\section{Sample inclusion}

112 One hundred and twelve EDTA whole blood samples, for which malaria examination was ordered 113 by the physicians, were collected at the Erasmus MC University Medical Center in Rotterdam, the

114 Netherlands, between December 2019 and December 2020, including the follow-up samples of 115 patients after initiation of treatment. In addition, 32 samples from asymptomatic, healthy 116 individuals with no suspicion of malaria were collected for the determination of the Limit of Blank 
117 (LoB). Samples that were older than $24 \mathrm{~h}$ post collection or had a volume of less than $500 \mu \mathrm{L}$ were

118 excluded. To investigate which RBC abnormalities perturb the MI-RBC examination, blood

119 samples were selected from routine hematology of patients that were not suspected of malaria, 120 comprised a high number of $\mathrm{NRBC}(>5 \%)$ and a relatively normal WBC count of $<20\left(\mathrm{x} 10^{3} / \mu \mathrm{L}\right)$.

121 NRBC was used as the primary selection criterion as these frequently co-exist with other RBC

122 abnormalities. With these selection criteria, 920 blood samples were examined with a high amount

123 of NRBCs, reticulocytes and/or morphological RBC abnormalities from patients with thalassemia,

124 sickle cell disease without hemato-oncological diseases, and ICU patients with infections and 125 stress erythropoiesis.

\section{Malaria examination reference methods}

128 In our hospital the standard procedure to diagnose malaria in freshly collected EDTA-blood 129 specimens involves an ICT RDT for malaria antigens and microscopic examination of both a QBC 130 capillary and stained thick and thin blood films. The rapid diagnostic antigen test (Binax NOW® 131 Malaria Test Binax, Inc. Maine, USA) and the QBC analyses were performed according to the 132 manufacturer's instructions. QBC capillaries were examined independently by two technicians by 133 microscopic analysis of two complete rows of the region between the bottom of the capillary and 134 the polynuclear leukocyte layer using an Olympus BX-60 fluorescence microscope equipped with 135 UV-filter, 50x objective and $12.5 \times$ oculars (total magnification $625 \times$ ). Thick blood films were 136 stained with Field's stain (Waldeck GMBH \& CO KG, Münster, Germany) and thin blood films 137 were fixed with absolute methanol for three minutes and subsequently stained with Diff Quick 138 (RAL Diagnostics, Martillac, France). Both staining procedures had been optimized for optimal 139 staining of Plasmodium parasites as well as Schüffner's dots and Maurer's clefts in infected 
140 erythrocytes. Thick and thin films were examined with regular light microscopes at a total

141 magnification of 1,250x. For all Plasmodium positive blood specimens the Plasmodium species

142 was confirmed by a real-time PCR method based on the method of Shokoples et al. ${ }^{19}$

\section{Flow cytometry analysis}

145 The XN-31 was operated in the Low Malaria (LM) mode, as this mode uses a three times higher

146 sample volume compared to other modes, which lowers the detection limit and thus increases the

147 sensitivity of the method. Using this Low Malaria mode of analysis, the XN-31 provides a 148 complete blood count (CBC), a qualitative result (positive, negative or indeterminate for malaria-

149 infected red blood cells, MI-RBC), a quantitative result (an absolute MI-RBC count and the 150 percentage of Plasmodium infected RBC) and a result for the suspected Plasmodium species (P.

151 falciparum or $P$. other, i.e. non-falciparum, or $P$. unknown). Speciation provided by $\mathrm{XN}-31$ is a 152 suspect flag approved for research use only purposes by the manufacturer. All study samples were 153 also processed on the Sysmex XN-1000 Series analyzer to collect full profile data (complete blood 154 count, $\mathrm{CBC}$ ), white blood cell differential (WDF), and reticulocytes (RET). The XN-31 results for 155 malaria were compared to those of the combined results of RDT, QBC and rt-PCR analyses in 156 order to determine the negative predictive value (NPV), positive predictive value (PPV), and 157 efficiency. The XN-31 MI-RBC count includes all malaria-infected RBCs, irrespective of life 158 stage. The differentiation into sexual (gametocytes) and non-sexual (rings, mature trophozoites, 159 schizonts) are research use only parameters. The quantification of infected erythrocytes on the XN16031 for both sexual and asexual stages of Plasmodium parasites were compared to the counting 161 results obtained by microscopic examination of thin and thick blood films. 
163 The LoB assessment was based on 32 malaria-negative blood samples from healthy individuals

164 with no symptoms and no suspicion for malaria. These samples had CBC values on the Sysmex

$165 \mathrm{XN}-1000$ hemocytometry analyzer within the reference ranges used in our department. The LoB

166 was calculated using the following formula: $\mathrm{LoB}=$ mean blank + 1.645(1SD of blank sample).

167

168 The LoD was determined using in vitro cultured RBC infected with $P$. falciparum NF54 parasites

169 (a generous gift of Dr. M. McCall, Radboudumc, Nijmegen, The Netherlands), that were serially

170 diluted in freshly collected full blood of a healthy donor. The LoD was calculated by the following

171 formula; $\mathrm{LoD}=\mathrm{LoB}+1.645$ (1SD of the sample with the lowest MI-RBC concentration above

172 the LoB with a reproducible qualitative test result).

173

174 The LoQ was calculated from the same dilution series and based on the point in the dilution series

175 that exceeded a coefficient of variation (CV) of $20 \%$. Quantification of infected RBC was also 176 determined by microscopic examination of thick and thin blood films. Thin blood films were used

177 to count infected erythrocytes if the parasitemia was above 4783 parasites per $\mu \mathrm{L}$ and thick blood 178 films were used to quantify parasites for all dilutions with a lower parasitemia.

$180 \quad$ RBC abnormality interferences on XN-31

181 In addition to a positive or negative result for $\mathrm{MI}-\mathrm{RBC}$, the $\mathrm{XN}-31$ can also provide an 182 indeterminate result. Blood films were made for all these XN-31 examined samples to confirm the 183 presence of RBC morphologies. The XN-31 scattergrams of all these samples were compared to 184 true positive Plasmodium samples in order to investigate the potential causing interference. The 185 selected blood samples were examined by the XN-31 hemocytometer and thin blood films were 
186 prepared and examined to confirm the presence of RBC morphologies. The XN-31 scattergrams

187 of all these samples were compared to true positive Plasmodium samples in order to investigate 188 the potential causing interference.

190 Statistical analysis

191 Data analysis was performed by using Analyse-it for Microsoft Excel version 2.30 and Microsoft 192 Excel 2016. Passing-Bablok regression analysis was used to determine accuracy of the MI-RBC 193 produced by the $\mathrm{XN}-31$ compared to microscopy.

\section{RESULTS}

196 Performance of the $\mathrm{XN}-31$ in clinical practice

197 Due to the COVID-19 pandemic and the drop in international travelers during the time of this

198 study, the number of requests for malaria examination was substantially decreased compared to 199 the pre-COVID-19 period. There were 112 included and 14 of them contained Plasmodium 200 parasites based on the results of analysis by RDT, QBC, thin and thick blood film examination and 201 rt-PCR. These 14 positive samples were derived from 8 patients, because 6 samples were follow202 up samples after initiation of malaria treatment. The 8 malaria patients were infected with $P$. 203 falciparum $(\mathrm{n}=6), P$. malariae $(\mathrm{n}=1)$ or $P$. vivax $(\mathrm{n}=1)$. Of the 112 samples, 109 were correctly 204 diagnosed by the $X N-31$, either as negative $(n=96)$ or positive $(n=13)$ and for those the 205 Plasmodium species were correctly determined as $P$. falciparum or $P$. other. The XN-31 produced 206 an indeterminate result for the remaining three samples, and therefore, the $\mathrm{XN}-31$ had a positive 207 and negative predictive value of $100 \%$ within an efficiency of $96 \%$ (Table 1). Of the three samples 208 for which an indeterminate result was reported, one sample contained $P$. malariae. The parasitemia 
209 in this sample appeared to be very low as only sporadically infected erythrocytes were found in

210 thick and thin blood films. In addition, this sample was also determined to contain microfilaria of

211 Mansonella perstans. The other two samples that had an indeterminate XN-31 result did not

212 contain Plasmodium infected RBC.

213able 1: Performance of XN-31 compared to the combination of parasitological examinations used in routine 214 patient care

215

\begin{tabular}{|c|c|c|c|}
\hline \multicolumn{4}{|c|}{ Overall Result of Parasitological Examinations } \\
\hline \multirow{3}{*}{$\begin{array}{c}\text { XN-31 } \\
\text { Result }\end{array}$} & Positive & Positive & Negative \\
\cline { 2 - 4 } & Indeterminate & 13 & 0 \\
\cline { 2 - 4 } & Negative & 1 & 2 \\
\cline { 2 - 4 } & Total & 14 & 96 \\
\hline \multicolumn{3}{|c|}{ Predictive Value and Efficiency } \\
\hline \multicolumn{2}{|c|}{ PPV } & NPV & 98 \\
\hline \multicolumn{2}{|c|}{$100 \%$} & $100 \%$ & Efficiency \\
\hline
\end{tabular}

216 PPV, positive predictive value, defines the probability of having Plasmodium in a sample with a 217 positive result. NPV, negative predictive value, describes the probability of not having 218 Plasmodium in a sample with a negative test result. Efficiency is the proportion of correctly 219 classified samples as negative or positive among all samples.

221 To investigate the correlation between the parasitemia determination by the $\mathrm{XN}-31$ and 222 microscopic examination, Passing-Bablok analysis was performed on 12 of the 14 positive 223 samples. For one positive sample the parasitemia could not reliably be determined by microscopy 224 because the patient was treated for malaria long enough that the parasite morphology in infected 225 erythrocytes was too aberrant to be reliably determined. In addition, in the P. malariae sample too 226 few infected erythrocytes were present that the parasitemia could not be accurately be determined 227 by microscopy. On the 12 remaining positive samples, Passing-Bablok analysis was performed 228 with the parasitemia results from the $\mathrm{XN}-31$ and the parasitemia determined in thin and thick blood 229 films to determine the accuracy of the $\mathrm{XN}-31$ across a range of distinct concentrations of 
Plasmodium infected RBC. The parasitemia of the XN-31 correlated well with the parasitemia

231 determined by microscopy (Figure 1) with a proportional bias of 21\% (y-intercept of 39.75 and

232 slope of 0.79). In addition, quantification of Plasmodium infected RBC in a dilution series prepared

233 from in vitro cultured RBC infected with P. falciparum in freshly collected blood of a healthy

234 donor, demonstrated a good agreement between the parasitemia determined by the XN-31 and

235 microscopic methods as well as a clear linear response with a best fit line of $y=440.7+1.12 x$

236 (Figure 2).

\section{Determination of $\mathbf{X N - 3 1}$ detection limits for Plasmodium infected erythrocytes}

239 As shown in Table 2 the LoB was determined to be 4.7 infected $\mathrm{RBC} / \mu \mathrm{L}$ and the LoD was

240 determined to be 5.9 infected $\mathrm{RBC} / \mu \mathrm{L}$. In addition, a dilution series of in vitro cultured $P$.

241 falciparum was used to determine the LoQ. As shown in Figure 3 the lowest concentration at

242 which the $\% \mathrm{CV}$ was still below $20 \%$ was $19 / \mu \mathrm{L}$, which had a CV of $19 \%$.

Table 2: Determination of LoB and LoD for the XN-31

\begin{tabular}{|c|c|}
\hline \multicolumn{2}{|c|}{ Determination of LoB for MI-RBC\# $(/ \mu \mathbf{L})$} \\
\hline Mean \#MI-RBC of blanks (32 samples) & 2.4 \\
\hline 1SD & 1.4 \\
\hline LoB = mean blank + 1.645(1SD of blank sample) & 4.7 \\
\hline Determination of LoD for MI-RBC\# (/ $\mu \mathbf{L})$ & 4.9 \\
\hline Mean \#MI-RBC of Target LoD Sample (10 replicates) & 0.7 \\
\hline 1SD & 5.9 \\
\hline LoD = LoB + 1.645 (1SD of low conc. sample)
\end{tabular}

244 LoB was calculated using samples of patients not infected with $P$. falciparum. The LoD was 245 calculated based on the LoB and 1 SD of the dilution sample mean with a reproducible positive test result with the lowest number of MI-RBCs above the LoB. Abbreviations: LoB, limit of blank; LoD, limit of detection; SD, standard deviation. 
250 In order to examine which RBC abnormalities could trigger indeterminate results, 920 samples

251 from 254 unique patients with RBC abnormalities (ranging from 1 to 16 samples per patient) were

252 selected from regular patient care hemocytometry. Included samples contained no Plasmodium

253 parasites, had greater than 5\% NRBC's and less than $20 \times 10^{9} \mathrm{WBC} / \mathrm{L}$, or came from the hematology

254 clinic. Processing of these 920 blood samples on the XN-31 generated 449 negative, 429

255 indeterminate and 42 false positive results for the detection of Plasmodium infected RBC. The MI-

256 RBC values of these false positive samples varied substantially as a wide range of 20 to 44,310

257 infected $\mathrm{RBC} / \mu \mathrm{L}$ was observed. This result demonstrates that the false positive results did not only

258 occur with low MI-RBC values being incorrectly detected. Table 3 shows the frequency of

259 occurrence of specific RBC abnormalities (as determined by the $\mathrm{XN}-1000$ ) in samples classified

260 by $\mathrm{XN}-31$ as positive, negative or indeterminate for MI-RBC qualitative judgment. Upon

261 reviewing the results for these samples on the XN-1000, more than $67 \%$ of the samples with an

262 MI-RBC false positive result and $62 \%$ of the samples with indeterminate result had greater than

$26310 \%$ NRBCs. It is clear in Table 3 that there are lower percentages of samples with increased

264 NRBCs and reticulocytes that are triggering the false positive MI_RBC results. There were also

265 samples with lower numbers of NRBC and reticulocytes triggered an indeterminate or false

266 positive MI-RBC result. Therefore, thin blood films of all false positive MI-RBC samples, were

267 manually re-evaluated by two trained microscopists to examine the abnormal RBC morphology

268 present in these samples. In total 42 samples from 31 patients gave false positive MI-RBC results.

269 Of these, $15 / 42$ samples (36\%) were from 8 sickle cell disease patients with sickle cells present in

270 their blood films. In the examined group of abnormal RBC samples especially the sickle cell

271 patients triggered many a false positive or an indeterminate MI-RBC result and never a negative

272 result apart from the few samples from sickle cell patients for which no sickle cells in their blood 
273 films could be found. Furthermore false positive or indeterminate MI-RBC results were also

274 generated with the samples from premature newborns (6/42), having high numbers of reticulocytes

275 and NRBC counts. Other patient groups and RBC anomalies occurring in the false positive MI-

$276 \mathrm{RBC}$ result group were hemochromatosis (8/42), beta thalassemia (12/42), leukemia/lymphoma

277 (6/42), and sepsis (1/42). Many patients in the false positive MI-RBC group had multiple diagnoses

278 and had multiple samples taken at different time points. Many patients in the false positive MI-

279 RBC group had multiple diagnoses, notable all hemochromatosis samples came from a single

280 patient with beta thalassaemia.

281 Table 3: Detection of Plasmodium infected RBCs in routine blood samples with RBC abnormalities using the $\mathrm{XN}-31$ analyzer

\begin{tabular}{|l|c|c|c|}
\hline & \multicolumn{2}{|c|}{ XN-31 Result for the detection of Plasmodium infected RBCs } \\
\hline $\begin{array}{l}\text { Sysmex XN-1000 Parameter (\# } \\
\text { out of 920) }\end{array}$ & $\begin{array}{c}\text { NEGATIVE }(\% \text { out of } \\
920)\end{array}$ & $\begin{array}{c}\text { INDETERMINATE (\% out } \\
\text { of 920) }\end{array}$ & $\begin{array}{c}\text { POSITIVE (\% out } \\
\text { of 920) }\end{array}$ \\
\hline NRBC >10\% (404) & $111(12 \%)$ & $265(29 \%)$ & $28(3 \%)$ \\
\hline RET >1.5\% (699) & $251(27 \%)$ & $411(45 \%)$ & $37(4 \%)$ \\
\hline RBC ABN Flag (448) & $105(11 \%)$ & $313(34 \%)$ & $30(3 \%)$ \\
\hline RET ABN SCAT (251) & $22(2 \%)$ & $205(34 \%)$ & $24(3 \%)$ \\
\hline & 489 & 1194 & 119 \\
\hline
\end{tabular}

284 Most samples included comprise more than a single abnormality.

285 Abbreviations: NRBC, nucleated red blood cell; RET, reticulocytes; WBC ABN, white blood cell

286 abnormal; RBC ABN, red blood cell abnormal; RET ABN SCAT, reticulocyte abnormal

287 scattergram.

288

289 To determine whether a true positive MI-RBC sample can be distinguished from a false positive

290 MI-RBC sample, we compared the MI-RBC scattergrams of the XN-31 analyses. In Figure 4, a

291 representative example of a true positive MI-RBC sample and a false positive sample are shown.

292 The events in the forward scatter light and side-fluorescent light (FSC and SFL) scattergram of a

293 true positive MI-RBC sample demonstrate a compact and perpendicular pattern with the defined 
294 clusters of RBC infected by one or multiple ring forms as explained by Pillay et al. ${ }^{12}$ (shown in

295 the green circles in Figure 4A). On the other-hand the scattergrams of false positive MI-RBC

296 samples demonstrate a dispersed pattern at a $45^{\circ} \mathrm{C}$ angle (located within the orange circle) (Figure

297 4B).

298

\section{DISCUSSION}

300 Our study shows that the XN-31 can be used in clinical practice as a fast and easy screening assay

301 for malaria that provides reliable qualitative and quantitative results. Therefore, $\mathrm{XN}-31$ can easily

302 be integrated in regular 24/7 patient care diagnostics settings in non-endemic counties and can

303 provide all required information to clinicians to timely start proper treatment. Compared to other

304 screening assays, the $\mathrm{XN}-31$ is the only test that can provide rapid results $(<1$ minute) to determine

305 the presence of Plasmodium infected RBC with a detection limit equivalent to thick blood film

306 examination $^{5}$ in combination with a Plasmodium species differentiation and an accurate

307 parasitemia quantification. The $\mathrm{XN}-31$ can accurately determine the parasitemia in samples with

308 low numbers of infected erythrocytes, as the limit of detection and quantification was determined

309 to be 5.9 and 19 infected RBC per $\mu \mathrm{L}$, respectively. Thereby the detection limit of the $\mathrm{XN}-31$ is

310 equivalent to thick and thin blood examination which has on average a detection limit of $\sim 10$

311 parasites per $\mu \mathrm{L} .^{5}$

312

313 Although we did not get any false positive or false negative results for samples of patient suspected

314 for malaria, it is known that submicroscopic malaria exists. ${ }^{20}$ In these cases the number of infected

315 erythrocytes is below the detection limit of thick blood film examination, and thus also below the

316 detection limit of the $\mathrm{XN}-31$. Therefore, false negative results can occur, but most submicroscopic 
317 malaria cases are asymptomatic and occur in patients from endemic areas with extensive immunity

318 against malaria or in patients infected with a benign, non-falciparum, Plasmodium species that will

319 present with a typical and characteristic fever pattern returning every 48 or 72 hours. ${ }^{21-23}$ Hence,

320 for patients whom malaria is clinically suspected, but a negative result is obtained, regardless of

321 which test is used, repeat testing should be undertaken periodically. In specific cases further

322 examinations by more sensitive methods can be indicated for patients for which a negative result

323 by the $\mathrm{XN}-31$ has been generated. In our analytical performance evaluation we achieved an LoD

324 of $\sim 6 \mathrm{MI}-\mathrm{RBC} / \mu \mathrm{L}$, which is significantly lower than the $20 \mathrm{MI}-\mathrm{RBC} / \mu \mathrm{L}$ cut-off set for qualitative

325 judgment of MI-RBC present or absent. The threshold for defining a sample as positive could

326 therefore possibly be adjusted by the manufacturer.

327

328 Next to the hypothetical possibility of false negative results due to patients with very low 329 parasitemia, our study demonstrated that indeterminate and false positive MI-RBC results can 330 occur as well. Examination of a large panel of blood samples of patients with a variety of RBC 331 abnormalities demonstrated not only a high frequency of indeterminate results ( 50\%), but also 332 false positive results $(\sim 5 \%)$. These false positive MI-RBC results were predominantly encountered 333 for blood samples from sickle cell patients and patients with increased numbers of nucleated 334 erythrocytes and/or reticulocytes. Conditions of stressed erythropoiesis such as may occur in 335 thalassemia or other hemoglobinopathies are mentioned in the instructions for use as potentially 336 giving an erroneous MI-RBC positive result. Analysis of the scattergrams demonstrated substantial 337 differences between the true positive and false positive MI-RBC samples, and therefore, future 338 refinement of the automatic interpretation script of the $\mathrm{XN}-31$ for the obtained scattergrams should 339 result in improved performance of the $\mathrm{XN}-31$. Thus, caution is required for patients with abnormal 
340 blood cell morphology and review of the scattergram is advised when authorizing results. It should

341 however be noted that in our study we deliberately enriched the number of samples measured with

342 those expected to cause interferences and that the actual occurrence of such issues may be

343 substantially lower in the routine setting where only samples from patients suspected to have

344 malaria will be measured on $\mathrm{XN}-31$.

346 In order to better determine what could produce indeterminate and false positive MI-RBC results

347 by the XN-31, abnormal RBC samples were examined. For detection of MI-RBC, the software

348 determines the number of events in the M-gating area as shown in Figure 4. If the number of

349 detected events exceeds a certain threshold an indeterminate or positive result will be generated

350 according to clustering patterns defined by algorithms. When interfering cell types are present that

351 produce a scattergram with a distinct cluster of particles in the M-gating area, the algorithm will

352 override the presence of generalized background scatter, producing a false positive MI-RBC result.

353 This also means that when 20 or more parasites/ $\mu \mathrm{L}$ are present, but no distinct cluster can be

354 detected due to interference, an indeterminate result will be produced that is marked with an

355 abnormal scattergram flag. Examination of the panel of abnormal RBC samples showed that a

356 number of diseases and conditions frequently occurred in both the false positive and indeterminate

357 MI-RBC results group; beta thalassemia, leukemia, lymphoma, premature newborns, and sickle

358 cell disease. The observation occurring most frequently for the false positive samples was sickle

359 cell disease in crisis. Although there were no sickle cell patients included in the study population

360 for suspected Plasmodium infection, it can be hypothesized that a patient with sickle cell disease

361 and malaria should get a valid MI-RBC present result (cluster detected) although the actual

362 parasitemia value would be overestimated. Premature newborns, beta thalassemia and 
363 hemochromatosis are associated with stressed or disturbed erythropoiesis as well and we speculate

364 that triggering of indeterminate and false positive results is highly correlated with diseases and

365 conditions associated with the presence of immature cells in the erythrocyte lineage or with

366 severely abnormal RBC morphology.

367

368 Although in our study population of patients suspected for a Plasmodium infection, none of the

369 mentioned diseases were present, it is very well possible as RBC abnormalities occur relatively

370 frequently in the population in malaria endemic areas. ${ }^{24}$ Some of these abnormalities can even

371 cause mortality in malaria patients making it even more important to understand exactly which

372 RBC abnormalities cause indeterminate or positive result on the XN-31. Mortality in sickle cell

373 patients with malaria is a problem in endemic countries that have a high prevalence of sickle cell

374 disease. More than $80 \%$ of people that have sickle cell disease live in sub-Saharan Africa where

375 most Plasmodium deaths occur. ${ }^{25}$ In one study from Cameroon, it was found that 2 out of every

37610 sickle cell patients who died had malaria. ${ }^{26}$ To date, there have been preliminary studies done

377 how interferences affect the results of the $\mathrm{XN}-31$, but there is some discrepancy in the results and

378 what conditions can trigger an abnormal scattergram on the $\mathrm{XN}-31 .{ }^{14,15}$ Further need to be done in

379 order to determine whether the XN-31 can properly detect Plasmodium infected RBC in patients

380 with sickle cell disease and other diseases that significantly affect red blood cell morphology

381 and/or erythropoiesis.

383 When an indeterminate result occurs, it is clear that additional examinations by other methods are 384 required to confirm whether Plasmodium infected RBC are present or not to prevent the reporting 385 of a false positive MI-RBC result, which could lead to misdiagnosis. To mitigate the possibility of 
386 having a false positive MI-RBC result with potentially serious consequences, we recommend to

387 evaluate the hemocytometric scattergrams to determine whether or not there is distinct cluster

388 formation of particles within the M-gating area where parasites are detected, as seen in a true

389 positive Plasmodium sample. In case of abnormal RBC morphology and a positive malaria result,

390 Plasmodium infected RBC should be confirmed by microscopic examination of thick and/or thin

391 blood films. However the scattergrams of true positive and false positive MI-RBC samples are

392 different and therefore further refinement of XN-31 gating and interpretation algorithms should be

393 able to increase the specificity.

395 Finally, there are some limitations of this study. Firstly, the number of included patients for 396 diagnostic accuracy was smaller than anticipated due to travel restrictions during the COVID-19

397 outbreak. Secondly, the LoD and LoQ studies were performed using P. falciparum parasites 398 cultured in vitro in $\mathrm{RBC}$ as there were no patient samples with a parasitemia high enough to dilute 399 serially across the linear range. Although the use of in vitro cultures on the XN-31 is not approved 400 in the specifications, our results showed that no interferences were present if the dilution series is 401 prepared by dilution of P. falciparum parasites cultured in RBC in vitro in freshly collected blood 402 of a healthy donor. Using this method accurate results were obtained for the LoB, LoD, and LoQ.

\section{CONCLUSION}

405 The $\mathrm{XN}-31$ is a promising alternative for rapid diagnostic antigen tests, as mutations in the HRP-

4062 gene will not interfere with the accuracy of results on the XN-31. However, our study 407 demonstrated that false positive results can occur in sickle cell patients or other RBC abnormalities 408 such as elevated NRBCs and/or reticulocytes and confirmation by the reference method is 
409 necessary. However, the scattergrams of true positive and false positive MI-RBC samples are

410 different and therefore further refinement of XN-31 gating and interpretation algorithms should be

411 able to increase the specificity. And until that has been achieved, caution is required for patients

412 with abnormal blood cell morphology. Ultimately, we showed that the $\mathrm{XN}-31$ can be a fast and

413 accurate screening method for the detection and quantification of Plasmodium infected RBC in

414 blood samples of patients suspected for malaria.

415

416 DECLARATIONS

417 Ethics approval

418 Ethical clearance for the use of residual blood samples for scientific purposes was granted by the 419 Medical Ethics Review Board of the Erasmus MC, University Medical Center Rotterdam, the 420 Netherlands (MEC 2012-047).

\section{Consent for publication}

423 Not applicable.

425 Availability of data and materials

426 The data that support the findings of this study are available from Erasmus MC, Department of 427 Clinical Chemistry but restrictions apply to the availability of these data, which were used under 428 license for the current study, and so are not publicly available. Data are however available from 429 the authors upon reasonable request and with permission of Erasmus MC, Department of Clinical 430 Chemistry. 
433 The authors declare that they have no competing interests.

$435 \quad$ Funding

436 Sysmex Europe GMBH provided free of charge reagents for the study. No monetary payments

437 were made to any of the investigators.

439 Authors' contributions

$440 \mathrm{TK}, \mathrm{YB}, \mathrm{RK}, \mathrm{JH}$, and HR all contributed to the study design, study execution, interpretation of the 441 data, and substantially revised the drafts. TK performed the statistical analysis and drafted the 442 work.

443

\section{Acknowledgements}

445 We thank Dr. Matthew McCall, Rianne Stoter and Marga van de Vegte-Bolmer for providing 446 samples of in vitro cultured Plasmodium falciparum NF54 parasites in RBC.

\section{REFERENCES}

449 1. World Health Organization. World Malaria Report: 20 Years of Global Progress and $450 \quad$ Challenges. Geneva: WHO; 2020.

451 https://www.who.int/publications/i/item/9789240015791. Accessed November 9, 2021.

452 2. Snow RW. Global malaria eradication and the importance of Plasmodium falciparum

453 epidemiology in Africa. BMC Med. 2015;13(1):14-16. doi:10.1186/s12916-014-0254-7

454 3. World Health Organization. Malaria: diagnostic testing. 2016:1-3.

455 http://www.who.int/csr/resources/publications/drugresist/malaria.pdf. 
456 4. CDC. Malaria Diagnostic Tests. Malaria Diagnostic Tests.

457 https://www.cdc.gov/malaria/diagnosis_treatment/diagnostic_tools.html\#tabs-1-1.

$458 \quad$ Published 2020. Accessed April 26, 2021.

459 5. Boonstra MB, Koelewijn R, Brienen EAT, et al. Malaria diagnosis in a malaria non-

460 endemic high-resource country: high variation of diagnostic strategy in clinical

461 laboratories in the Netherlands. Malar J. 2021;20(1):411. doi:10.1186/s12936-021-03889-

$462 \quad 7$

463 6. Mishra M, Misra R. Immunochromatographic Methods in Malaria Diagnosis. Med

464 Journal, Armed Forces India. 2007;63(2):127. doi:10.1016/S0377-1237(07)80054-2

465 7. Portero J-L, Rubio-Yuste M, Descalzo MA, et al. Accuracy of an

466 Immunochromatographic Diagnostic Test (ICT Malaria Combo Cassette Test) Compared

467 to Microscopy among under Five-Year-Old Children when Diagnosing Malaria in

468 Equatorial Guinea. Malar Res Treat. 2010;2010:1-6. doi:10.4061/2010/858427

469 8. Cheng Q, Gatton ML, Barnwell J, et al. Plasmodium falciparum parasites lacking

$470 \quad$ histidine-rich protein 2 and 3: A review and recommendations for accurate reporting.

$471 \quad$ Malar J. 2014;13(1):1-8. doi:10.1186/1475-2875-13-283

472 9. Charpentier E, Benichou E, Pagès A, et al. Performance evaluation of different strategies

473 based on microscopy techniques, rapid diagnostic test and molecular loop-mediated

474 isothermal amplification assay for the diagnosis of imported malaria. Clin Microbiol

$475 \quad$ Infect. 2020;26(1):115-121. doi:10.1016/j.cmi.2019.05.010

476 10. Ponce C, Kaczorowski F, Perpoint T, et al. Diagnostic accuracy of loop-mediated

477 isothermal amplification (LAMP) for screening patients with imported malaria in a non-

$478 \quad$ endemic setting. Parasite. 2017;24. doi:10.1051/parasite/2017054 
479 11. Pöschl B, Waneesorn J, Thekisoe O, Chutipongvivate S, Panagiotis K. Comparative 480 diagnosis of malaria infections by microscopy, nested PCR, and LAMP in Northern Thailand. Am J Trop Med Hyg. 2010;83(1):56-60. doi:10.4269/ajtmh.2010.09-0630

482 12. Pillay E, Khodaiji S, Bezuidenhout BC, Litshie M, Coetzer TL. Evaluation of automated malaria diagnosis using the Sysmex XN-30 analyser in a clinical setting. Malar J. 2019;18(1):15. doi:10.1186/s12936-019-2655-8

13. Tougan T, Suzuki Y, Itagaki S, et al. An automated haematology analyzer XN-30 distinguishes developmental stages of falciparum malaria parasite cultured in vitro. Malar

14. Zuluaga-Idárraga L, Rios A, Sierra-Cifuentes V, et al. Performance of the hematology J. 2018;17(1):1-8. doi:10.1186/s12936-018-2208-6 analyzer XN-31 prototype in the detection of Plasmodium infections in an endemic region of Colombia. Sci Rep. 2021;11(1):5268. doi:10.1038/s41598-021-84594-y

15. M'baya B, Mfune T, Samon A, Hwandih T, Münster M. Evaluation of the Sysmex XN-31 automated analyser for blood donor malaria screening at Malawi Blood Transfusion Services. Vox Sang. 2021;(June):1-8. doi:10.1111/vox.13208

16. Post A, Kaboré B, Reuling IJ, et al. The XN-30 hematology analyzer for rapid sensitive

497 17. Toya Y, Tougan T, Horii T, Uchihashi K. Lysercell M enhances the detection of stage498 specific Plasmodium-infected red blood cells in the automated hematology analyzer XN49931 prototype. Parasitol Int. 2021;80(October 2020):102206.

$500 \quad$ doi:10.1016/j.parint.2020.102206

501 18. Tougan T, Toya Y, Uchihashi K, Horii T. Application of the automated haematology 
analyzer XN-30 for discovery and development of anti-malarial drugs. Malar J. 2019;18(1):1-12. doi:10.1186/s12936-019-2642-0

504 19. Shokoples SE, Ndao M, Kowalewska-Grochowska K, Yanow SK. Multiplexed real-time 505 PCR assay for discrimination of Plasmodium species with improved sensitivity for mixed infections. J Clin Microbiol. 2009;47(4):975-980. doi:10.1128/JCM.01858-08

507 20. Pousibet-Puerto J, Cabezas-Fernández MT, Lozano-Serrano AB, et al. Submicroscopic Malaria in Migrants from Sub-Saharan Africa, Spain. Emerg Infect Dis. 2019;25(2):349. doi:10.3201/EID2502.180717

510 21. T B, L O, I F, C D. Asymptomatic malaria infections: detectability, transmissibility and public health relevance. Nat Rev Microbiol. 2014;12(12):833-840. doi:10.1038/NRMICRO3364

22. EE B, JJ van $\mathrm{H}, \mathrm{PJ}$ van $\mathrm{G}$, et al. A case report of transfusion-transmitted Plasmodium malariae from an asymptomatic non-immune traveller. Malar J. 2013;12(1).

23. A W, AW D, I H, et al. High Rates of Asymptomatic, Sub-microscopic Plasmodium vivax Infection and Disappearing Plasmodium falciparum Malaria in an Area of Low Transmission in Solomon Islands. PLoS Negl Trop Dis. 2015;9(5). doi:10.1371/JOURNAL.PNTD.0003758

24. Min-Oo G, Gros P. Erythrocyte variants and the nature of their malaria protective effect. Cell Microbiol. 2005;7(6):753-763. doi:10.1111/J.1462-5822.2005.00524.X to Plasmodium falciparum in sickle cell trait erythrocytes is driven by oxygen-dependent growth inhibition. Proc Natl Acad Sci U S A. 2018;115(28):7350-7355. 
doi:10.1073/pnas.1804388115

526

26. Eleonore NLE, Cumber SN, Charlotte EE, et al. Malaria in patients with sickle cell anaemia: Burden, risk factors and outcome at the Laquintinie hospital, Cameroon. BMC Infect Dis. 2020;20(1):1-8. doi:10.1186/s12879-019-4757-x

\section{FIGURES}

Figure 1: Accuracy of XN-31 parasitemia determination in clinical patient samples compared to parasitemia determined by microscopy

Figure 2: Accuracy of XN-31 for dilution series of in vitro cultured $P$. falciparum compared to microscopy A linear dilution series was prepared of in vitro cultured P. falciparum infected RBC in freshly obtained blood of a healthy donor, after which the parasitemia was determined by $\mathrm{XN}-31$ and microscopic examination of blood films. Line of best fit is in red comparing the results of the $\mathrm{XN}$ $31 \mathrm{MI}-\mathrm{RBC} \#(/ \mu \mathrm{L})$ to the microscopy results.

\section{Figure 3: Determination of Limit of Quantification (LoQ) of the XN-31}

Each point in the graph represents the mean of 10 replicates in that concentration and the $\% \mathrm{CV}$ associated with those replicates. The LoQ is the lowest concentration of infected erythrocytes with a $\% \mathrm{CV}<20$ (indicated with the red line), which is in this case an MI-RBC of 19 parasites $/ \mu \mathrm{L}$. Abbreviations: LoQ, limit of quantification; $\mathrm{CV}$, coefficient of variation.

Figure 4: Comparison of XN-31 scattergrams of a true positive Plasmodium falciparum sample with a false-positive MI-RBC sample

Figure $\mathbf{A}$ is a scattergram of a true positive MI-RBC samples containing erythrocytes infected with Plasmodium falciparum. Figure B is a scattergram of a sample of a patient in sickle crisis that produced a false positive MI-RBC result by the XN-31. The red particles are what the XN-31 suspects to be a Plasmodium infected red blood cell, the teal particles are the leukocytes, and the dark blue particles are the non-infected red blood cells or debris. In Figure A, a true positive sample, the cluster of events is vertical (green circles), whereas the false-positive events identified in the MI-RBC channel in the sickle cell crises samples cluster at a $45^{\circ}$ angle (orange circle in panel B).

Abbreviations: FSC, forward scatter light; SFL, side-fluorescent 
Figures

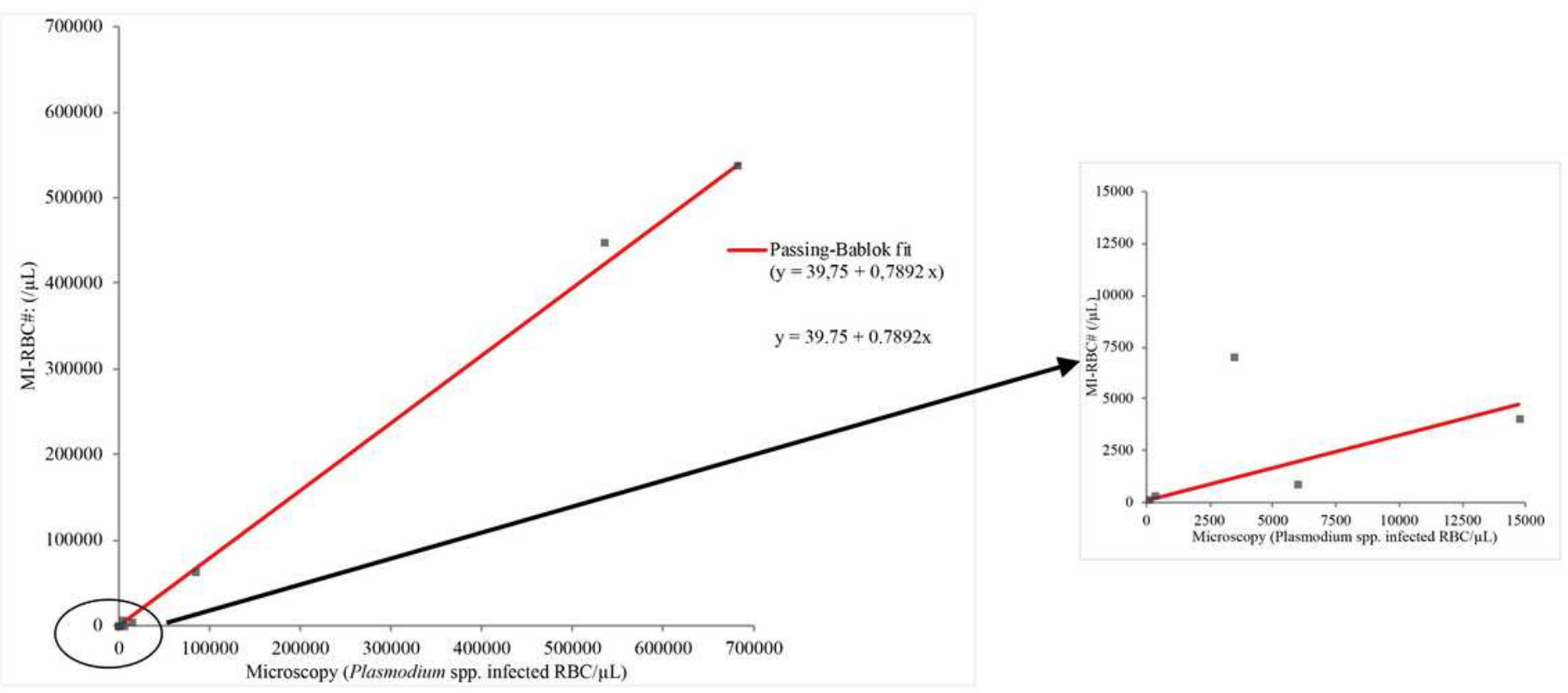

\section{Figure 1}

Accuracy of $\mathrm{XN}-31$ parasitemia determination in clinical patient samples compared to parasitemia determined by microscopy

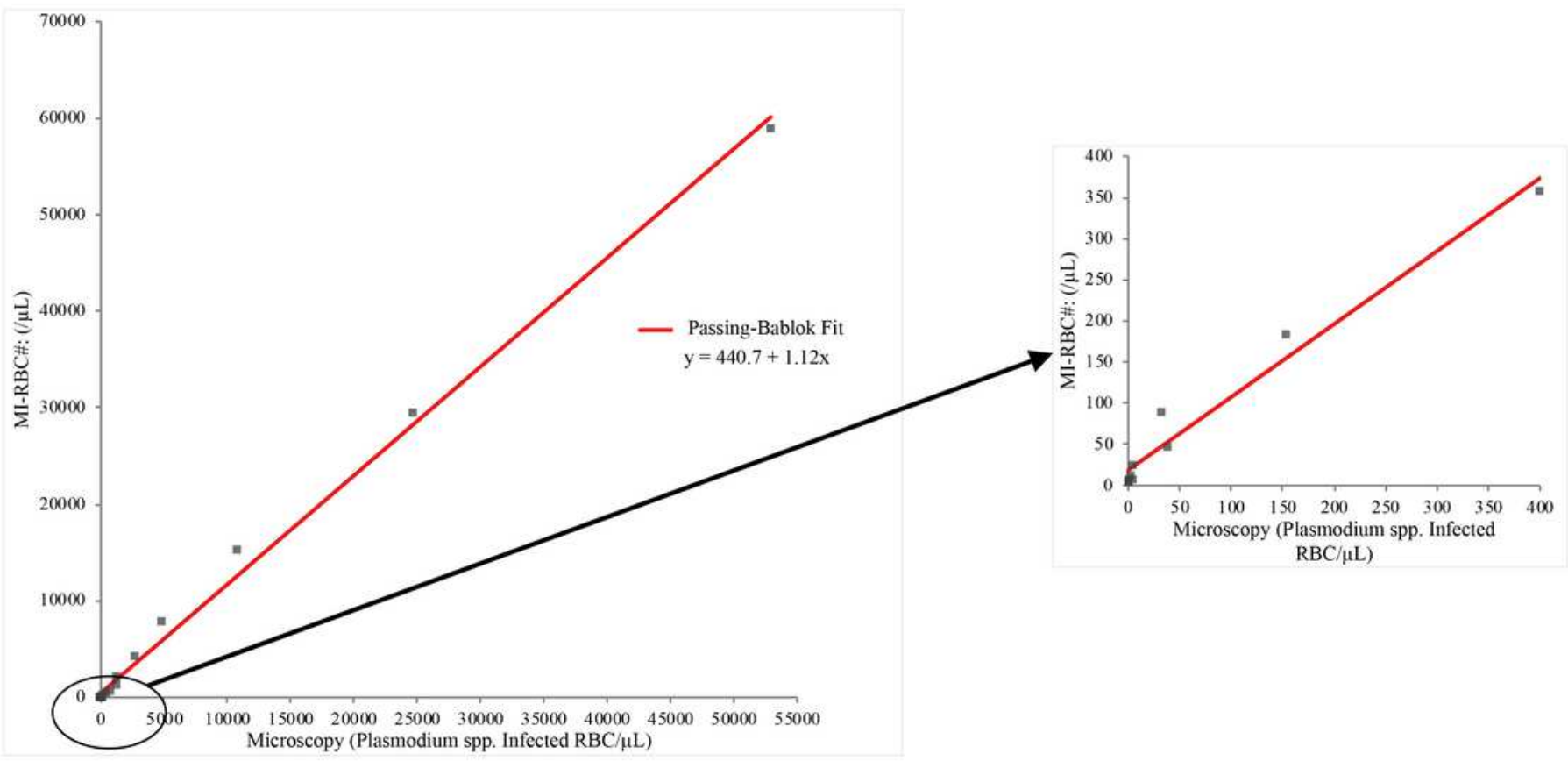

Figure 2 
Accuracy of $\mathrm{XN}-31$ for dilution series of in vitro cultured $\mathrm{P}$. falciparum compared to microscopy $\mathrm{A}$ linear dilution series was prepared of in vitro cultured P. falciparum infected RBC in freshly obtained blood of a healthy donor, after which the parasitemia was determined by XN-31 and microscopic examination of blood films. Line of best fit is in red comparing the results of the $\mathrm{XN}-31 \mathrm{MI}-\mathrm{RBC} \#(/ \mu \mathrm{L})$ to the microscopy results.

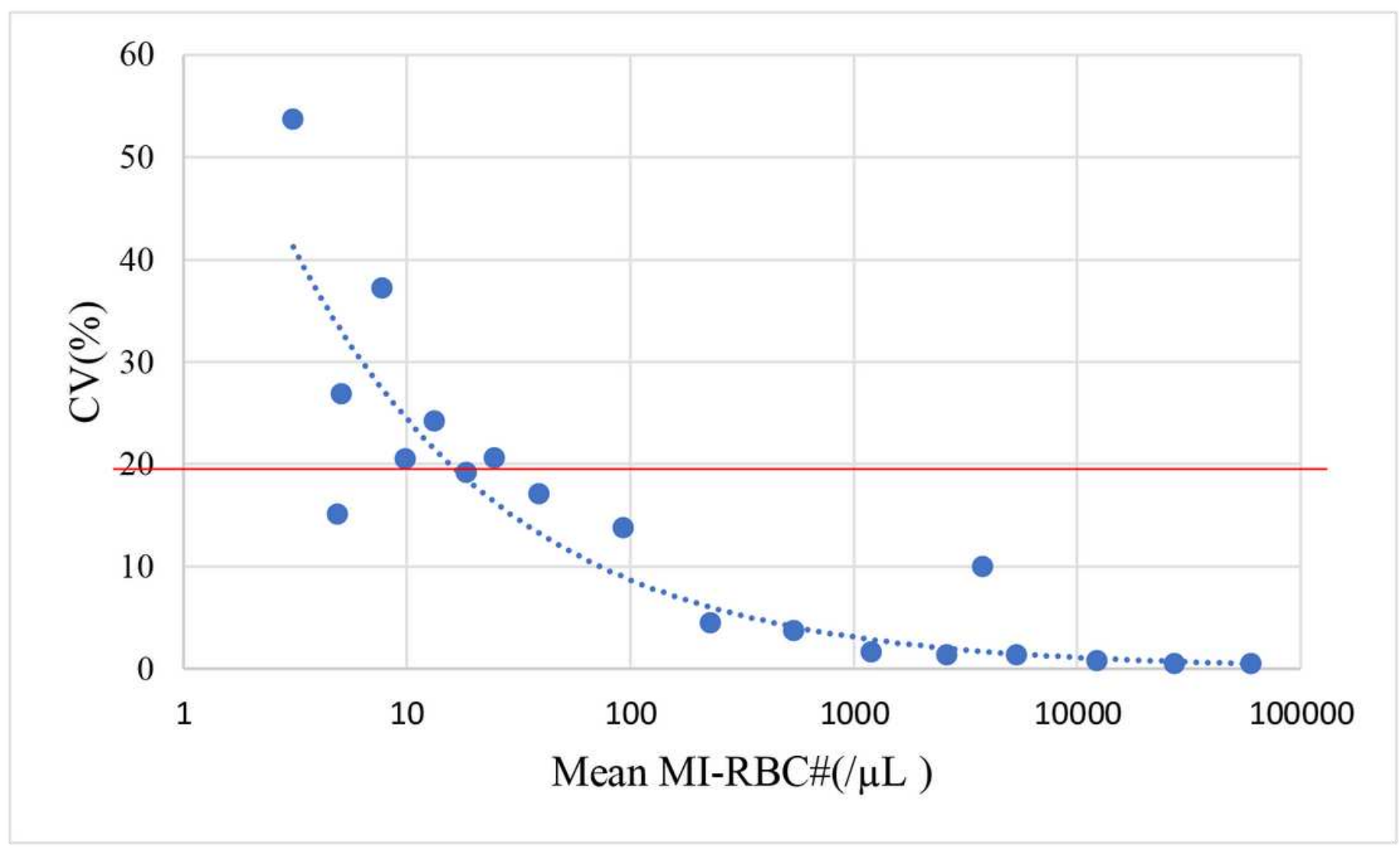

\section{Figure 3}

Determination of Limit of Quantification (LoQ) of the XN-31 Each point in the graph represents the mean of 10 replicates in that concentration and the \%CV associated with those replicates. The LoQ is the lowest concentration of infected erythrocytes with a \%CV $<20$ (indicated with the red line), which is in this case an MI-RBC of 19 parasites/ $\mu \mathrm{L}$. Abbreviations: LoQ, limit of quantification; CV, coefficient of variation 
A.

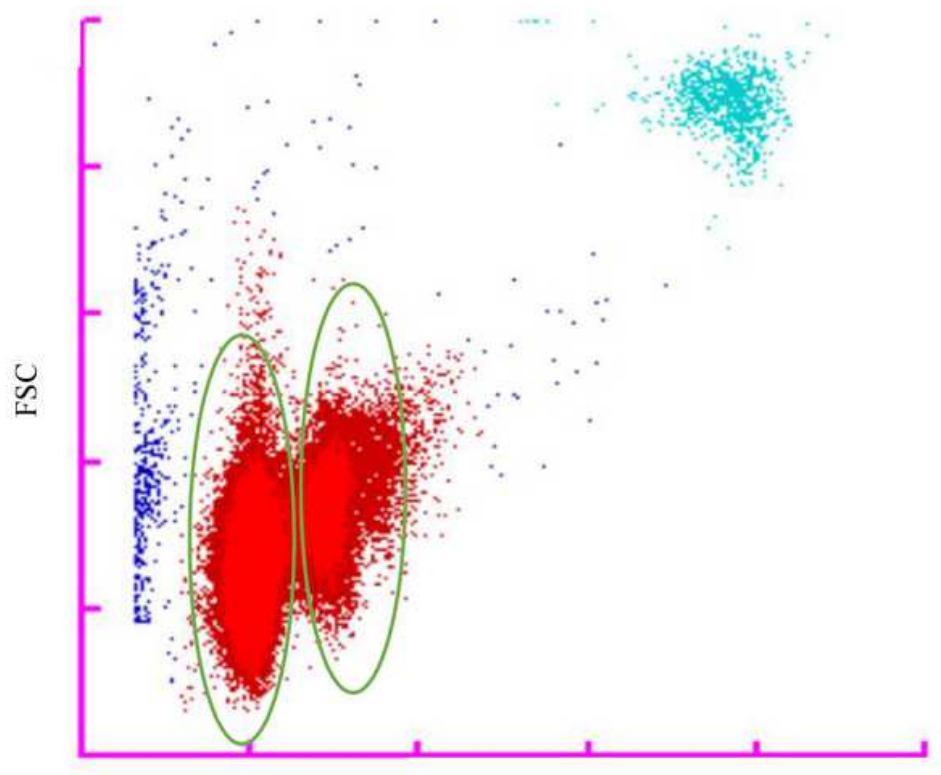

SFL
B.

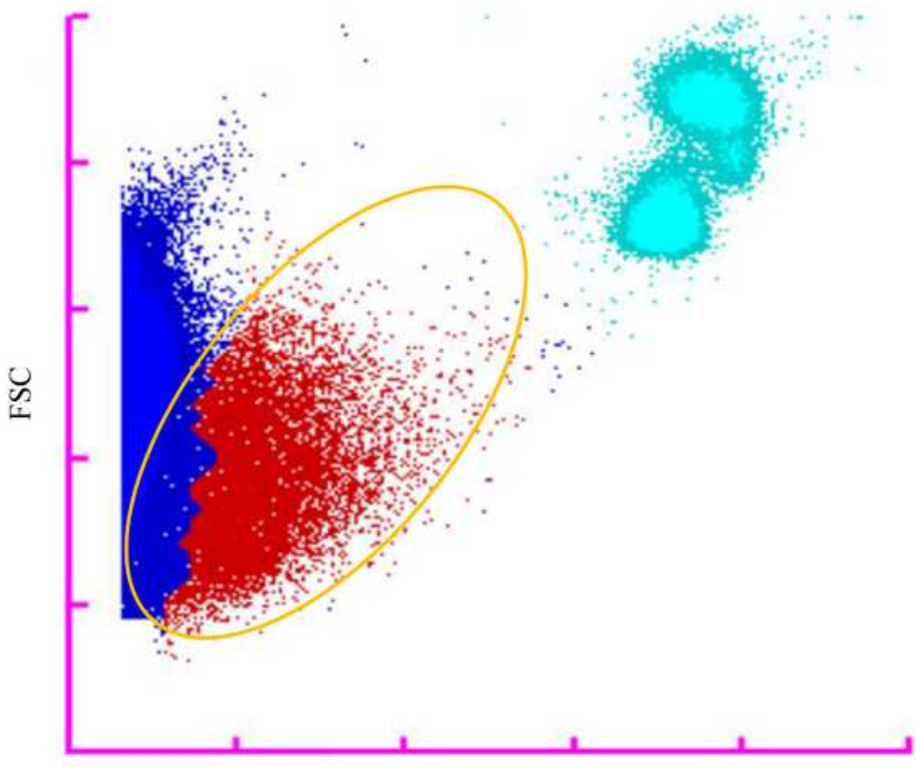

SFL

\section{Figure 4}

Comparison of $\mathrm{XN}-31$ scattergrams of a true positive Plasmodium falciparum sample with a falsepositive MI-RBC sample

Figure $A$ is a scattergram of a true positive MI-RBC samples containing erythrocytes infected with Plasmodium falciparum. Figure $B$ is a scattergram of a sample of a patient in sickle crisis that produced a false positive MI-RBC result by the XN-31. The red particles are what the $\mathrm{XN}-31$

suspects to be a Plasmodium infected red blood cell, the teal particles are the leukocytes, and the dark blue particles are the non-infected red blood cells or debris. In Figure A, a true positive sample, the cluster of events is vertical (green circles), whereas the false-positive events identified

in the MI-RBC channel in the sickle cell crises samples cluster at a $45^{\circ}$ angle (orange circle in panel B).

Abbreviations: FSC, forward scatter light; SFL, side-fluorescent 